\title{
Philosophiques
}

\section{Les énoncés psychologiques à la première personne et le problème de la connaissance de soi}

\section{Michel Seymour}

Volume 18, numéro 1, printemps 1991

URI : https://id.erudit.org/iderudit/027139ar

DOI : https://doi.org/10.7202/027139ar

Aller au sommaire du numéro

Éditeur(s)

Société de philosophie du Québec

ISSN

0316-2923 (imprimé)

1492-1391 (numérique)

Découvrir la revue

Citer cet article

Seymour, M. (1991). Les énoncés psychologiques à la première personne et le problème de la connaissance de soi. Philosophiques, 18(1), 3-23.

https://doi.org/10.7202/027139ar
Résumé de l'article

Un argument en faveur de l'infaillibilité a priori est considéré puis rejeté. Il s'agit de critiquer un argument de type cartésien visant à fonder la connaissance de soi sur un type privilégié de rapport épistémique. On montre que plusieurs catégories d'énoncés psychologiques à la première personne ne peuvent donner lieu à un tel savoir infaillible. La discussion culmine sur l'examen du Cogito. Il est démontré que le philosophe cartésien ne peut savoir a priori qu'il pense puisqu'il ne peut savoir a priori que chaque inscription en lui de la forme verbale " Je pense " constitue un acte intentionnel de penser. 
PHILOSOPHIQUeS, Vol. XVIII, Numéro 1, Printemps 1991

\title{
ARTICLES \\ LES ÉNONCÉS PSYCHOLOGIQUES À LA PREMIÈRE PERSONNE ET LE PROBLÈME DE LA CONNAISSANCE DE SOI*
}

\author{
par Michel Seymour
}

RÉSUMÉ. Un argument en faveur de l'infaillibilité a priori est considéré puis rejeté. Il s'agit de critiquer un argument de type cartésien visant à fonder la connaissance de soi sur un type privilégié de rapport épistémique. On montre que plusieurs catégories d'énoncés psychologiques à la première personne ne peuvent donner lieu à un tel savoir infaillible. La discussion culmine sur l'examen du Cogito. Il est démontré que le philosophe cartésien ne peut savoir a priori qu'il pense puisqu'il ne peut savoir a priori que chaque inscription en lui de la forme verbale «Je pense * constitue un acte intentionnel de penser.

ABSTRACT, An argument favourable to a priori infallibility is considered and then rejected. Specifically, it is a cartesian argument which purports to justify infallible self-knowledge on the basis of a special epistemic access. Many first person psychological sentences are shown to be defective in this respect. The discussion culminates with an examination of the Cogito. It is shown that the cartesian philosopher cannot claim to know a priori that he is thinking since he does not know a priori that all inscriptions in himself of the verbal form * I think * constitute an intentional act of thinking.

* Le texte qui suit a été pour une large part suscité à l'occasion du séminaire donné par Tyler Burge au printemps 90 a UCLA. Je lui dois par conséquent beaucoup meme si la critique « wittgensteinienne * que je propose résulte de mes propres reflexions sur le sujet. 
La discussion au sujet de l'individuation des contenus de pensée en philosophie de l'esprit a conduit récemment certains philosophes à se demander s'il est possible de soutenir de façon cohérente à la fois l'anti-individualisme et le principe cartésien de l'autorité de la première personne. (Par exemple, Davidson 1987; voir aussi Burge 1988 et Davidson 1988). L'anti-individualisme implique que les contenus de pensée sont individués au moins en partie par l'environnement physique ou l'environnement social. L'individualisme n'implique, au contraire, aucune détermination de ce genre et c'est pourquoi il semble, à première vue, qu'il soit le seul à pouvoir s'accommoder de l'idée que l'agent a pleinement accès à ses propres contenus de pensée. En effet, si les environnements social ou physique interviennent dans l'individuation des contenus de pensée, comment l'agent peut-il avoir autorité sur ses propres contenus de pensée? Plusieurs philosophes s'entendent quand même pour dire que les deux doctrines sont compatibles. D'autres en profitent pour questionner le principe de l'autorité de la première personne (Millikan 1984). Mais le débat ouvre la voie à un examen renouvelé des thèses concernant le caractère infaillible de la connaissance de soi. Je voudrais montrer que même si le sujet individuel a, jusqu'à un certain point, autorité sur certaines de ses attitudes psychologiques, cette autorité ne saurait s'expliquer par le fait qu'il a un accès épistémique privilégié à ses propres états "internes". Je ne vais pas tenter ici de fournir une explication de l'autorité de la première personne. Je veux discuter plutôt d'un argument de type "cartésien " et lui opposer une critique "wittgensteinienne». L'anti-individualisme est certes compatible avec l'autorité de la première personne, mais il n'y a pas grand chose à dire en faveur de la thèse "cartésienne" selon laquelle il existerait une connaissance de soi infaillible a priori et dont le fondement serait épistémique (Descartes 1964, Vol. 7, 23-24). Cette thèse pose cependant suffisamment d'énigmes pour mériter un examen indépendant.

Il convient de s'arrêter un instant sur la notion d'a prioricité invoquée ici. Par connaissance a priori, je n'entends pas seulement une connaissance acquise à partir de l'appréhension de principes universels (vérités logiques, définitions). Je reconnais qu'il peut $y$ avoir a prioricité même lorsque le savoir dont il est question concerne un fait 
particulier. Corrélativement, j'admets aussi la possibilité de l'a priori contingent. En troisième lieu, je suis aussi disposé à admettre, pour les fins de la discussion, que la connaissance a priori puisse avoir elle-même parfois un caractère événementiel et je n'impose pas la condition qu'elle doive consister en des états mentaux d'appréhension quasi in temporels dont les conditions d'individuation ne feraient pas appel à des séquences d'événements mentaux. Pour qu'on puisse parler de vérité a priori, l'essentiel est que l'agent n'ait pas besoin de se sortir de la pensée dans laquelle il se trouve pour accéder à cette vérité. De cette manière, on reconnaît qu'un agent puisse avoir une connaissance $a$ priori de l'événement mental intentionnel dans lequel il se trouve. Comme on le verra plus loin, toute la question sera de savoir si l'existence d'événements mentaux intentionnels peut elle-même être établie a priori. Cela reste à discuter mais je ne veux pas, à ce stade-ci, écarter cette éventualité. Voilà la raison pour laquelle j'autorise en principe qu'on puisse parler de connaissance a priori dès lors que l'agent peut accéder à cette connaissance sans sortir de la pensée singulière dans laquelle il se trouve. Si on pouvait déduire de principes généraux l'existence d'événements mentaux intentionnels, il se pourrait alors que certains d'entre eux aient un caractère réflexif. Les conditions de vérité des énoncés qui rapportent l'existence de tels événements se. raient satisfaites pourvu que ces énoncés eux-mêmes fassent l'objet d'une pensée. Ils exemplifieraient alors une connaissance $a$ priori au sens où je l'entends.

On peut, au départ, énumérer les conditions qui doivent être satisfaites pour qu'un énoncé psychologique à la première personne puisse donner lieu à un savoir, non pas indubitable, mais " infaillible " et a priori. Il s'agit de conditions que doit satisfaire un argument de type cartésien. Je n'entends pas produire un argument contre toute infaillibilité a priori (et encore moins a posteriori bien que, dans ce dernier cas, il me semble que la réfutation soit plus facile) et je concentre plutôt mes efforts sur un argument particulier qui suppose une relation de transparence épistémique entre le sujet et ses propres événements mentaux. Voici donc ces conditions :

- Condition illocutoire: l'énoncé doit être disponible pour un acte de jugement ou, à tout le moins, permettre de rapporter ou décrire le phénomène psychologique en question. En somme, il doit être susceptible de vérité ou de fausseté. 
- Condition pbénoménologique : ce qui est rapporté ou décrit par l'énoncé doit être appréhendé consciemment et avoir un caractère événementiel.

- Condition épistémologique : L'agent doit avoir un accès a priori au phénomène et ne pas faire reposer la vérité de l'énoncé faisant l'objet de son jugement sur une évidence empirique comme la mémoire par exemple. Cette condition peut sembler contredire la précédente dans la mesure où un événement mental doit en apparence quand même faire l'objet d'une expérience, mais j'emploie ici la notion d'a prioricité dans le sens que j'ai décrit plus haut qui autorise à parler de connaissance $a$ priori dans ce cas. Il suffit que l'agent accède *de l'intérieur * à la connaissance de cet événement.

- Condition ontologique: les contours du phénomène rapporté doivent être bien définis.

- Condition logique : les concepts exprimés pour le caractériser doivent être clairs et distincts.

- Condition sémantique: Le phénomène doit être rapporté par l'énoncé sur le mode de dicto et non de re et servir à spécifier un contenu intentionnel et non un rôle fonctionnel.

On notera qu'en formulant ces conditions, $j$ 'ai pris pour acquis que le jugement ou la pensée de l'agent s'articule au travers d'un medium langagier et on pourrait objecter que je suis, de cette manière, en train de me donner une prémisse suffisante pour prendre en défaut le cartésien. Mais je ne présuppose une théorie citationnelle que pour faciliter la discussion. Si jamais cette présupposition risquait de nuire aux arguments cartésiens, je la retirerais aussitôt. Il faut dire aussi qu'une théorie citationnelle ne peut à elle seule suffire, de toute façon, à réfuter le cartésien. Une telle théorie est compatible avec l'idée que les constituants linguistiques appartiennent au langage de la pensée.

Les conditions phénoménologique et sémantique ne retiendront pas beaucoup notre attention. La première permet d'éliminer d'emblée les énoncés qui rapportent des états psychologiques ou des sentiments. On tient pour évident le fait que, la plupart du temps, les états psychologiques et les sentiments ne peuvent pas faire l'objet d'une connaissance infaillible. La condition sémantique permet elle aussi d'éliminer des candidats indésirables. Les énoncés rapportant des attitudes de re, au sens classique (par oppo- 
sition aux attitudes de se (Lewis 1979) ou à celles qui font intervenir des sens de re (McDowell 1984), et les énoncés au sujet d'états fonctionnels ne peuvent, trivialement, donner lieu à un savoir infaillible. Cette dernière condition est violée lorsque, par exemple, l'énoncé psychologique met en rapport l'attitude avec un événement ou objet qui en a été la cause.

Mais on peut s'interroger sur la pertinence d'introduire les conditions logique et ontologique. Ne sont-elles pas trop difficiles à satisfaire? La connaissance de soi infaillible ne repose-t-elle pas, justement, sur le fait qu'elle porte sur des concepts ou des objets dont l'identité est restée indéterminée? Il faut rappeler ici encore une fois qu'il s'agit de discuter un argument de type cartésien. Les contraintes logique et ontologique que j'impose sont d'ailleurs des contraintes analogues à celles imposées par Descartes luimême. Il faut dire aussi que ce sont là des contraintes fort naturelles dans la mesure où l'on prend généralement pour acquis qu'une chose ne peut exister que si elle a des conditions d'individuation déterminées. Dans une telle perspective, une connaissance dirigée sur des objets ou des concepts dont les contours sont indéterminés est une connaissance sans objet et ne peut donc pas accéder au statut de connaissance. Je suis loin d'être d'accord avec le point de vue selon lequel on devrait rejeter les concepts et objets vagues de notre ontologie, mais je reconnais volontiers que le fardeau de la preuve repose sur les épaules du philosophe qui veut les admettre. Si les candidats au savoir infaillible font intervenir des objets et concepts flous, c'est, jusqu'à nouvel ordre, une raison pour ne pas leur accorder le statut de "savoirs". Quoiqu'il en soit, les deux contraintes en question n'affectent pas le cour de notre argument qui concerne le Cogito cartésien.

Les différentes conditions que j'ai introduites nous permettent d'éliminer différentes catégories d'énoncés. Je vais rapidement considérer les possibilités qui s'offrent à nous. On pourrait penser, par exemple, que des énoncés comme

(1) J'ai mal

(2) J'ai peur

(3) J'ai froid 
sont des candidats idéaux au savoir infaillible, mais il n'est pas clair qu'ils satisfont la condition illocutoire. Comme on le verra plus loin, mon point de vue critique s'inscrit à l'intérieur d'un cadre d'analyse wittgensteinien et $j$ 'adopte par conséquent une sémantique des conditions d'assertabilité. Même si les énoncés considérés ont des conditions de vérité, leur signification dépend de conditions d'assertabilité. Ils sont très souvent utilisés pour accomplir des actes illocutoires expressifs. Ainsi compris, ils ne sont pas disponibles pour la vérité ou la fausseté. Les catégories épistémologiques de doute et de certitude ne trouvent donc pas d'application. (Wittgenstein 1953, \#246, \#258, \#288, \#290 ; p. 221)

On peut être tenté de rétorquer que l'on peut se tromper en énonçant de telles phrases. Il y a des cas très clairs où l'agent dit qu'il a mal alors que cela n'est pas le cas. Les hypochondriaques ne prouvent-ils pas que les énoncés rapportant la douleur peuvent être vrais ou faux et qu'ils servent à articuler des jugements sur la douleur? Il faut dire tout de suite que la position de Wittgenstein ne se veut pas générale et peut autoriser des exceptions. Mais la plupart des exemples d' $\mathrm{derreurs}^{*}$ au sujet de la douleur que l'on ressent s'expliquent par le fait que l'agent n'est pas correctement "branché * sur les événements psychologiques qui l'habitent. L'agent utilise toujours l'énoncé pour exprimer sincèrement sa douleur, mais il existe une interférence entre l'état qu'il ressent en apparence et l'état qu'il ressent en réalité. L'idée de Wittgenstein est que, la plupart du temps, les énoncés rapportant la douleur sont utilisés pour accomplir des actes illocutoires expressifs. À moins d'être affecté d'un tempérament flegmatique extrême ou d'être un partisan farouche du stoïcisme, les locuteurs n'entretiennent pas, en général, une distanciation épistémique à l'égard de la douleur. Cela n'interdit pas des circonstances plus ou moins exceptionnelles, mais cela donne le modèle général à partir duquel analyser les énoncés qui font référence à la douleur.

\section{III}

Ce modèle ne peut pas, bien entendu, s'appliquer à tous les cas. Les énoncés qui rapportent des sensations ont en général des conditions d'assertabilité qui satisfont la condition illocutoire. On pense en particulier aux énoncés qui rapportent des expériences perceptuelles comme : 
(4) Je vois une tache de couleur rouge

(5) Je sens une odeur d'estragon

(6) Je goûte la saveur d'un cabernet sauvignon

(7) Je sens la rugosité du bois

(8) J'entends le bruit d'un tam-tam

Tous ces énoncés violent, à vrai dire, la condition sémantique. Ils permettent à l'agent de se prononcer sur les "objets " matériels de l'expérience perceptuelle, c'est-à-dire sur ce qui est susceptible d'en constituer la cause. Mais faisons l'hypothèse que ces phrases peuvent être réécrites de telle façon qu'elles mettent l'agent en rapport avec des "sensations-de-rouge ", des "sensations d'estragon ", etc. On essaie ici pour les besoins de la discussion d'avantager autant que possible le partisan de l'infaillibilité. On fait donc la supposition que les compléments d'objet directs ont dans ces énoncés, une fois reformulés, une occurrence oblique et satisfont ainsi la condition sémantique.

La question se pose quand même de savoir s'ils satisfont pleinement la condition ontologique. Les sensations ontelles des contours bien définis ? On peut confronter ceux qui répondent par l'affirmative au paradoxe de la transitivité des sensations de couleurs. Supposons, par exemple, des types de sensations de couleur $\mathrm{A}$, B et $\mathrm{C}$ et des occurrences de sensations $a, b$ et $c$ qui en sont les instances. Considérons ensuite l'argument suivant :

(i) $\mathrm{Si} \mathrm{A}=\mathrm{B}$ et $\mathrm{B}=\mathrm{C}$, alors $\mathrm{A}=\mathrm{C}$

(Transitivité de l'identité pour les sensations de couleur)

(ii) a est indiscernable de $b$ (par hypothèse)

(iii) b est indiscernable de $c$ (par hypothèse)

(iv) a est discernable de $c$ (par hypothèse)

(v) Deux * occurrences * de sensation, de couleur sont (Critère d'identité pour les sensations de couleurs)

les mêmes sensations de couleur si et seulement si elles sont indiscernables (quant à la couleur)

(vi) $A=C$

(de (iv) et (v))

(vii) $\mathrm{A}=\mathrm{B}, \mathrm{B}=\mathrm{C}$ (de (ii) (iii) et (v))

(viii) $\mathrm{A}=\mathrm{C}$ (de (i) et (vii))

(ix) (viii) contredit (vi)

On peut penser que le problème découle du critère d'identité des sensations. Goodman, par exemple, pense que le critère devrait être remplacé par le suivant : 
(v)' Deux sensations de couleur sont identiques si chacune forme, pour un agent donné, des paires indiscernables avec les mêmes sensations de couleur. (Goodman 1951, 96-98; 196)

L'hypothèse de Goodman est qu'il doit ne pas exister une sensation de couleur $c$ discernable de a et indiscernable de b (ou, à l'inverse, indiscernable de a et discernable de b) pour que a soit identique à $b$. Or c'est précisément ce qui se produit dans les prémisses (ii), (iii) et (iv). Si on remplace (v) par (v)', on ne peut plus inférer (vii) et, par conséquent, (viii) non plus. On bloque de cette façon la conclusion indésirée (ix).

Mais la plupart des philosophes s'entendent au contraire pour localiser le problème au niveau de la prémisse (i). On suppose que la transitivité de l'identité s'applique aux sensations, mais cela implique qu'elles peuvent entrer dans des énoncés d'identité. Cela présuppose une réification injustifiée des sensations. L'idée est que les sensations ne sont pas des "choses" au même titre que les objets physiques. Si on nie l'existence des "sense data", les relations de similarité, de ressemblance ou d'indiscernabilité peuvent s'appliquer, mais non la relation d'identité proprement dite. Voilà pourquoi c'est la prémisse (i) de l'argument qui est problématique. La prémisse (iv) ne l'est pas. On accepte que, si les «sense data» existent, alors le critère d'identité proposé est le bon. Le problème est seulement que les «sense data" n'existent pas. C'est la solution adoptée par la plupart des philosophes. (Armstrong 1979, Dummett 1979, Wright 1975 et Peacocke 1981, par exemple). Mais si les «sense data* n'existent pas, les énoncés qui les rapportent ne peuvent avoir des conditions de vérité déterminées. Les jugements que l'on réalise à partir de ces énoncés peuvent alors difficilement ètre infaillibles au sens requis par un argument de type cartésien. Le problème est que les sensations n'ont pas des contours bien définis. En somme, on ne satisfait pas la condition ontologique.

Mais que dire du cas où l'on est mis en présence d'une odeur particulière pour la première fois, par exemple celle de l'estragon, puis invité à l'identifier aussitôt ? La condition susceptible de ne pas être satisfaite est, cette fois-ci, la condition épistémologique. Pour être en mesure d'identifier à nouveau correctement une odeur à laquelle on vient d'être confronté, il faut juger que c'est la même sensation et il faut alors immanquablement faire appel à la mémoire. Cela suffit pour qu'il soit impossible de parler ici d'infaillibilité 
a priori. L'agent fait reposer la justification de son jugement sur une condition indirecte. Le théoricien des sense data est donc confronté à une difficulté de principe. Il postule un espace mental d'objets et ne tient pas compte du fait que les sensations se profilent sur un arrière-fond toujours changeant. Autrement dit, elles s'inscrivent dans le temps. Telle est l'intuition de ceux qui refusent de réifier les sensations. Celles-ci ne peuvent être considérées comme des objets que si on les abstrait artificiellement du contexte dans lequel elles apparaissent et si l'on ne tient pas compte de la temporalité qui les affecte.

\section{IV}

Il faut alors préférablement se rabattre sur les jugements portant sur des contenus de pensée. Les énoncés de la forme

(9) Je pense que $p$

(10) Je constate que $p$

(11) Je juge que $p$

semblent être des candidats tout indiqués pour l'infaillibilité. Il faut supposer dans ces trois cas que les verbes spécifient des actes et non des états. Le problème, cette fois-ci, est que l'on ne peut être certain de satisfaire la condition logique selon laquelle nos concepts devraient être clairs et distincts. Un wittgensteinien se sent particulièrement à l'aise sur ce terrain. Les jugements qui rapportent nos contenus de pensée vont immanquablement faire intervenir des concepts. Or ceux-ci ont en général un caractère normatif et ont des règles d'interprétation associées. Ceci nous oblige à reconnaître la possibilité de l'erreur et nous permet de mettre en échec un argument en faveur de l'infaillibilité de la connaissance qui intervient dans l'affirmation des énoncés (9)-(11).

L'argument wittgensteinien peut être formulé comme suit. On veut admettre la possibilité de l'erreur dans l'application de la plupart de nos concepts et la raison en est que ceux-ci ont un caractère normatif. Une norme qui ne pourrait jamais être correctement appliquée ne serait pas une norme. Qui dit normativité dit qu'il existe une règle et que la fixation ou l'introduction de cette règle doit être distinguée de son application. Il est ensuite affirmé que cette distinction doit aller de pair, au niveau de l'apprentissage de la règle, avec la distinction entre son appréhension et la capacité de l'appliquer correctement. La compréhension 
d'une règle doit incorporer autant un savoir propositionnel qu'un savoir-faire. Mais il est alors difficile de ne pas reconnaître la possibilité logique qu'un gouffre puisse subsister entre le savoir propositionnel de la règle et son application. Il y a toujours de la place pour la formulation d'un doute sceptique. Une fois que le caractère normatif des concepts est admis, il est difficile de prétendre à l'infaillibilité dans nos jugements, puisqu'ils font intervenir des applications particulières d'une règle normative. (Kripke 1982)

Pour contourner cette difficulté, il faut invoquer la posibilité de concepts innés. On pourrait penser que les concepts de douleur ou de froideur, par exemple, sont des concepts innés. Il m'apparaît cependant clair que leur application est gouvernée au moins en partie par les normes en vigueur au sein d'une communauté. Il se peut que des communautés soient disposées plus facilement que d'autres à parler de "douleur". Cela révèle qu'il y a une normativité implicite. L'idée est que le concept de douleur est sans doute formé à partir d'expériences que l'agent entretient avec son environnement physique. Pour faciliter la communication, il a tendance à fixer ensuite les règles gouvernant l'application de ses concepts en se basant sur des expériences qu'il partage en commun avec le reste de la communauté. Si j'ai raison, cela montre à la fois le caractère acquis et non inné des concepts de douleur, ainsi que la normativité déterminée par la communauté. Par conséquent, même dans les cas exceptionnels où des énoncés rapportant la douleur sont utilisés pour la performance d'actes illocutoires assertifs, il n'y a pas d'infaillibilité puisque l'agent peut appliquer incorrectement le concept de douleur qu'il utilise en ne se conformant pas avec l'usage admis dans la communauté. Bien entendu, un agent peut utiliser des expressions sans se conformer, de cette manière, à l'usage qui est en vigueur au sein du groupe auquel il appartient. L'idée est seulement qu'en procédant de la sorte, il ne fait rien d'autre que d'essayer d'imposer de nouvelles normes.

\section{V}

Il nous reste cependant un autre recours, le Cogito cartésien. Ici, on évacue à première vue le problème de l'infaillibilité dans l'application des concepts, puisque l'énoncé que l'on considère fait seulement référence à l'activité de penser. En jargon phénoménologique, on dirait qu'il s'agit d'une pensée qui appréhende seulement l'aspect noé- 
tique et non le contenu noématique de nos actes de pensée. Les candidats à l'infaillibilité sont maintenant les énoncés :

(12) Par ce présent acte, je pense

OU

(13) Par ce présent acte, je suis en train de penser

ou

(14) À ce moment-ci, je pense

Je veux écarter immédiatement deux questions qui sont susceptibles de se poser. On peut se demander si la pensée que je pense peut être «individuée * de façon *individualiste ", c'est-à-dire individuée indépendamment de toute référence à l'environnemnt physique ou social. J'aurais tendance là aussi à penser que le terme *penser * a lui-même un contenu déterminé par les normes en vigueur au sein de la communauté. On peut douter que l'expression soit utilisée de la même façon chez les moines bouddhistes, en intelligence artificielle ou dans les écrits de John Searle. Mais je laisse ce point de côté. On peut aussi se poser la question de savoir si l'énoncé décrit le rapport qu'un sujet individuel, en tant que substance, entretient avec une action quelconque. Mais je laisse aussi cette question de côté. Dans la discussion qui précède et celle qui suit, il se peut que Lichtenberg ait raison et que le sujet-substance ne soit rien d'autre qu'une fiction de philosophe. Les problèmes que je veux soulever sont ailleurs.

Il semble qu'on ait enfin affaire aux candidats idéaux pour la raison suivante. Les cas qu'on a précédemment examinés manifestent l'absence de relations constitutives entre le point de vue et la topique (subject matter) et / ou entre l'acte accompli et l'acte représenté. Par exemple, les sensations font intervenir seulement un complément d'objet direct et sont des actes de perception alors que les attitudes qui les rapportent et que l'on considère comme des candidats à l'infaillibilité sont des jugements et leurs points de vue sont des contenus propositionnels entiers (par exemple, "que je vois une tache rouge "). Il semble qu'il $y$ ait maintenant une relation constitutive, d'une part, entre les deux actes et, d'autre part, entre le point de vue et la topique. Il y a une relation constitutive entre actes parce que les verbes dans les phrases (12), (13) et (14) ne doivent pas nécessairement être utilisés pour accomplir des actes de jugements. Les événements impliqués dans ces énoncés sont des actes de pensée, mais ils peuvent eux-mêmes sim- 
plement faire l'objet d'une pensée. Il y a aussi une relation constitutive entre le point de vue et la topique parce que la pensée porte sur elle-même. Cette relation constitutive est d'ailleurs ce qui permettrait de distinguer les arguments $a$ priori cartésiens des arguments a posteriori de Locke, qui font tous appel à un cil mental. (Locke 1959, Vol. 2, Book IV, Ch. IX, 305)

Pour bien comprendre ce qui se passe ici, il convient de considérer à nouveau des énoncés qui portent sur nos jugements. Comme on l'a vu, ces derniers ne peuvent être infaillibles à cause des concepts qui interviennent obliquement dans le rapport, mais on fait abstraction de cette difficulté. Pour le bénéfice de la discussion, on suppose que l'on peut juger que $p$ indépendamment du fait d'avoir un langage et on suppose aussi qu'aucune normativité n'intervient. On suppose en somme que les concepts invoqués sont innés. Considérons donc le cas d'énoncés comme

(15) Je juge que l'on ne peut nager dans du ciment sec

(16) Je doute qu'un texte clair puisse être généré par une pensée confuse

(17) J'infère que quelque chose est blanc du fait que la neige est blanche.

La position de type cartésien que je veux illustrer à l'occasion de ces énoncés implique les thèses suivantes :

(A) Ces pensées ont des conditions de vérité

(B) Leur signification est déterminée essentiellement par ces conditions de vérité. Elles n'auraient pas de signification si elles n'avaient pas de conditions de vérité. Il s'agit ici d'une application particulière de la sémantique selon laquelle les conditions de vérité d'un énoncé au mode indicatif constituent un ingrédient essentiel de sa signification conventionnelle.

(C) Les verbes «juger», *douter», et «inférer» servent à la fois à décrire un acte et à l'accomplir. En ayant la pensée que je juge que $p$, je performe l'acte de juger que $p$ et je le représente tout à la fois.

(D) Ces pensées voient leurs conditions de vérité satisfaites si et seulement si elles sont pensées et que l'agent s'engage cognitivement. Si l'agent juge l'énoncé (15), par exemple, cela est une condition nécessaire et suffisante pour la satisfaction de ses conditions de vérité. 
(E) Je peux alors infailliblement savoir a priori que ces pensées sont vraies.

L'idée est que, dans les exemples considérés, une occurrence de la pensée avec un engagement cognitif approprié entraîne aussitôt la satisfaction de ses conditions de vérité. Cela pourrait en soi se produire sans que l'agent s'en rende compte et c'est sans doute pour cette raison qu'on ne peut parler d'indubitabilité. Mais l'agent qui entretient cette pensée avec un engagement cognitif approprié se réfère à cette occurrence et est donc en mesure de savoir a priori qu'elle est vraie. Autrement dit, en vertu de (C), on établit l'existence d'une relation constitutive entre une pensée et ce sur quoi elle porte. À cause de cette relation constitutive, on établit avec (D) que, sous certaines conditions, les énoncés (15)-(17) ne peuvent être faux. Ils ne sont pas en soi incorrigibles et non révisables, mais ils le deviennent lorsque certaines conditions sont satisfaites. On obtient ainsi un savoir infaillible a priori.

La perspective wittgensteinienne que je propose pour les énoncés (15), (16) et (17) est la suivante. On accepte (A). Mais on rejette tout d'abord la thèse (B). On prétend plutôt que la signification conventionnelle est déterminée par des conditions d'assertabilité. Puis à l'encontre de la thèse $(C)$, on prétend que les énoncés (pensées) considérés ont des conditions d'assertabilité ambiguës. Ils peuvent être utilisés pour la performance de l'acte de juger ou pour rapporter cette performance. Autrement dit, en énonçant (pensant) "je juge que $p$ ", je peux faire au moins deux choses. Je peux ou bien accomplir l'acte de juger que $p$ ou rapporter l'existence d'un acte de juger que $p$. Mais je ne peux faire les deux à la fois.

Dans le cas où l'agent utilise l'énoncé pour rapporter un acte, son énoncé peut être vrai ou faux et satisfaire la condition illocutoire, mais n'est pas vrai a priori. Il ne satisfait pas la condition épistémologique. Par contre, dans le cas où l'agent accomplit un acte de juger, l'agent peut acquérir un savoir a priori. Par exemple, il sait a priori que, s'il utilise (15) comme un énoncé performatif, il rend vraies les conditions de vérité de l'énoncé. Mais, en tant que performatif, l'énoncé n'est pas disponible pour la vérité et la fausseté et ne peut pas, en lui-même, constituer un savoir. 
Ici c'est la condition illocutoire qui n'est pas satisfaite. L'action de juger ne peut être elle-même vraie ou fausse. Seul le contenu de l'acte peut raisonnablement être un support du prédicat de vérité.

Quelles raisons avons-nous de penser que les deux actes, celui de juger et celui de représenter l'acte de juger, ont lieu simultanément lorsqu'un agent entretient une pensée comme (15) avec un engagement cognitif approprié ? Le philosophe cartésien sera tenté de nous offrir une preuve par l'absurde. Que dire de la suggestion selon laquelle il peut servir seulement à décrire un jugement sans être un jugement? Selon lui, (15) est intuitivement vrai mais ne pourrait l'être s'il ne faisait que décrire. Puisqu'il est généralement utilisé pour décrire ce qui se passe au temps présent, le fait d'admettre qu'il ne s'agit pas d'un acte de juger revient à admettre que l'énoncé est faux. Mais on peut lui rétorquer que l'énoncé fait intervenir une référence à un certain intervalle de temps rapproché qui ne correspond pas à celui de son énonciation. Sans être lui-même un acte de juger, l'énoncé peut être vrai parce qu'il fait intervenir une référence à un événement autre que celui qui correspond à sa propre énonciation.

Que dire maintenant de l'hypothèse inverse? L'énonciation de (15) peut-elle constituer un acte de jugement sans du même coup être une description de l'acte de juger? Le cartésien peut s'appuyer ici sur une intuition en apparence très solide. Il faut distinguer le jugement appliqué à un énoncé «p" et le jugement appliqué à l'énoncé "Je juge que $p$ *. En prétendant que l'énonciation de "Je juge que $p$ * peut se réduire à l'acte de juger " $p$ ", ne sommes-nous pas en train de confondre les deux? La réponse est que nous n'avons pas confondu les deux. On reconnaît que l'énoncé (15) peut servir à décrire l'acte de jugement et que, utilisé de cette façon, il se différencie du jugement que $p$. On distingue donc nettement les deux sortes d'actes. La thèse est seulement que (15) peut être utilisé pour accomplir l'un ou l'autre. Lorsque (15) est utilisé comme un énoncé performatif, ses conditions de vérité sont satisfaites. Si l'agent juge ensuite que l'énoncé est vrai, il aura raison. Mais le fait que l'énoncé soit rendu vrai par l'énonciation n'implique pas que l'agent juge qu'il en est ainsi. L'erreur du cartésien est la suivante. Le verbe juger en (15) a des conditions de satisfaction quel que soit l'usage qui est fait de l'énoncé entier. À partir de là, il tire la conclusion erronée que le seul usage littéral possible de la phrase est de juger que ses 
conditions de vérité sont satisfaites. Il commet cette erreur parce qu'il supppose que la signification de l'énoncé est déterminée par ses conditions de vérité. Mais la signification de l'énoncé dépend d'abord et avant tout de ses conditions d'assertabilité et celles-ci sont ambiguës. Comme on l'a suggéré, il peut être utilisé pour affirmer l'existence de certaines conditions objectives de vérité ou pour * rendre vraies " ses conditions de vérité.

On peut donc réfuter l'argument du philosophe cartésien en s'en prenant à la prémisse (C). Mais il y a aussi le fait que la satisfaction des conditions de vérité de l'énoncé (15) requiert l'existence d'un engagement cognitif approprié et on peut se demander si la satisfaction de cette condition peut être connue a priori.

\section{VII}

Les mêmes remarques valent à peu près pour les énoncés (12)-(14) à cette exception près que leurs occurrences n'ont pas besoin d'être accompagnées d'un engagement cognitif approprié pour que leurs conditions de vérité soient satisfaites. Ils peuvent faire simplement l'objet d'une pensée, comme cela se produit lorsque l'on considère une hypothèse, on contemple une alternative ou on pose une question. Pour les énoncés (12)-(14), la thèse (D) peut être formulée plus fortement. La seule occurrence de ces énoncés (pensées) entraîne automatiquement la satisfaction de leurs conditions de vérité. On semble donc se rapprocher de la thèse d'infaillibilité cartésienne. Si l'agent peut avec l'expression démonstrative se référer à cette occurrence et si celle-ci ne peut que satisfaire les conditions de vérité de l'énoncé, comment peut-il ne pas savoir a priori que l'énoncé est vrai ? C'est ici que l'examen des démonstratifs s'avère indispensable.

La différence entre (12)-(14) et (15)-(17) est, comme on vient de le faire remarquer, qu'ils ne peuvent être faux même lorsqu'ils ne sont pas utilisés pour rapporter l'existence d'un acte de pensée. En tant que descriptions, ils satisfont la condition illocutoire. Puisqu'ils ne peuvent être faux, ne satisfont-ils pas aussi la condition épistémologique d'a prioricité ? La réponse est non. La réplique wittgensteinienne est tout d'abord que (12)-(14) ont aussi des conditions d'assertabilité ambiguës. Mais contrairement aux énoncés (15)-(17), l'ambiguïté n'est pas provoquée par la 
possibilité d'utiliser le verbe psychologique de différentes façons. Le responsable est cette fois-ci l'expression démonstrative. Les expressions démonstratives ont elles aussi des conditions d'utilisation qui peuvent varier d'un contexte à l'autre et qui peuvent servir à déterminer des conditions d'assertabilité distinctes dans ces contextes. Comme on le verra, l'erreur du cartésien est de supposer qu'un acte de démonstration d'une pensée peut être fait de l'intérieur de cette même pensée.

Les démonstratifs ont plusieurs usages. Ils peuvent servir à accomplir des actes de démonstration ou ils peuvent se comporter un peu comme des " pronoms paresseux * et être anaphoriquement liés à des descriptions définies. Or lorsqu'ils servent à accomplir des actes de démonstration, ils ne peuvent référer à la pensée dans laquelle ils apparaissent. Quand l'agent utilise de cette manière le démonstratif et qu'il affirme être en train d'avoir cette pensée-ci, on est tenté de répondre : laquelle? L'idée est que, dans cet usage, le démonstratif ne peut que référer à l'extérieur de la pensée dans laquelle il apparaît. Il fonctionne alors un peu comme un pistolet. Le contexte fournit les munitions et l'énonciation revient à presser sur la gachette. Supposer qu'une démonstration puisse être auto-référentielle équivaut à admettre un pistolet qui aurait la propriété de se viser luimême. Cela ne correspond à aucun usage réel.

Bien entendu, une expression démonstrative peut, à l'occasion, réaliser un acte d' "auto-référence». Un agent peut, à son insu, se pointer lui-même du doigt en désignant un individu dans un miroir ou il peut intentionnellement "se désigner lui-même* en pointant son propre corps. Mais dans tous ces cas, l'expression sert quand même à référer à quelque chose qui se situe à l'extérieur de la pensée.

Le second usage auquel je veux faire allusion concerne celui où l'expression est en quelque sorte une abréviation pour une description définie. Dans ce cas, il peut très bien y avoir auto-référence, mais cela dépend de la tournure des événements, c'est-à-dire de circonstances empiriques. Je peux alors, par exemple, être en train de penser cette pensée-ci, à savoir la pensée entretenue par $\mathrm{X}$ au temps $\mathrm{T}$ et au lieu $L$, et être en fait ce $X$ au temps T et au lieu L. Cela dépend des circonstances empiriques. En somme, je reconnais volontiers qu'un énoncé comme *Cette phrase-ci est en français» puisse faire intervenir une auto-référence. Il affirme que la phrase énoncée ici et maintenant est en français. Il s'agit là d'une affirmation tout à fait correcte et vraie. 
Mais il ne livre pas une information vraie a priori. L'information qu'il véhicule, relativement au contexte, a pour effet de rendre vraie son énonciation.

Ma conjecture au sujet des démonstratifs est donc la suivante. S'ils sont utilisés pour accomplir des démonstrations, ils vont sans doute de pair avec un mode de présentation épistémique, mais ils accomplissent une référence à l'extérieur de la pensée dans laquelle ils apparaissent et la condition d'a prioricité est violée. Le mode de présentation livre un savoir a posteriori. S'ils sont utilisés comme une abréviation pour une description définie, ils peuvent être auto-référentiels, mais c'est en vertu de circonstances empiriques. Encore une fois, la condition d'a prioricité est violée.

J'ai fait valoir que les démonstratifs avaient au moins deux usages. Dans le premier cas, ils ont un usage démonstratif «substantiel» alors que, dans l'autre, il s'agit d'un usage "dégénéré». Dans ce dernier cas, ils fonctionnent, elliptiquement ou par le biais d'une chaîne anaphorique, comme des abréviations de descriptions définies. Il y a donc au moins deux cas possibles et ils entraînent deux interprétations possibles du Cogito. Lorsque le démonstratif accomplit un acte de démonstration dans un énoncé utilisé pour décrire un acte mental, nous avons un énoncé vrai $a$ posteriori. Le démonstratif réfêre alors à une pensée extérieure à celle dans laquelle il apparaît.

Mais le deuxième usage est sans doute celui qui se rapproche le plus de l'hypothèse cartésienne. Dans ce cas particulier, une occurrence dégénérée du démonstratif est immédiatement suivie d'une description de l'acte de penser. Comme on l'a déjà fait remarquer, la seule occurrence d'une description de l'acte de penser implique la satisfaction des conditions de vérité de cette description. Le démonstratif permet ensuite de fixer la référence de la description. Une fois que l'on a mis en évidence la description définie dont il est une abréviation, la pensée décrite par l'énoncé est alors nulle autre que la pensée exprimée par ce même énoncé. Formulé de cette façon, le Cogito cartésien serait quelque chose comme :

(18) Par le présent acte (ici et maintenant), je pense

Ici on a à la fois l'exemple d'une auto-référence et la présence d'une occurrence qui suffirait à satisfaire ses conditions de vérité. Un énoncé de ce genre permet-il un savoir infaillible a priori ? On a déjà répondu que non en alléguant que le démonstratif ainsi conçu devenait auto-ré- 
férentiel seulement en vertu de circonstances empiriques. Mais n'est-il pas possible de s'en servir pour exprimer un savoir infaillible a priori ? On a peut-être pu établir que, d'une façon générale, l'énoncé servait à véhiculer un savoir a posteriori, mais cela n'interdit pas la possibilité d'une connaissance a priori à son sujet. On sait depuis Frege que pour qu'un énoncé soit qualifié d'a priori, il suffit qu'il soit possible de justifier sa vérité sans le secours de l'expérience sensible.

Pour répondre à la question, il faut déterminer si la satisfaction des conditions de vérité de l'énoncé peut, en tant que résultant de sa seule occurrence, faire l'objet d'un savoir a priori. Il faut dire qu'en lui-même, et donc indépendamment du fait d'être précédé d'une expression démonstrative, l'énoncé ne peut être jugé vrai a priori. Je peux être en train de faire autre chose que de penser. Mais le démonstratif permet de déterminer que la pensée dont il est question est celle qui est exprimée en (18). Il semble que je ne puisse penser (18) sans satisfaire ses conditions de vérité. En somme, (18) est vrai dans tous les contextes de pensée intentionnelle. La question est donc celle-ci : sait-on a priori que toute inscription mentale de (18) rend vrai le contenu propositionnel? Ia question n'est pas de déterminer si cela est indubitable, mais plutôt de déterminer si cela requiert le recours à l'observation ou s'il s'agit d'une connexion logique.

La question est une variante de celle-ci : existe-t-il une connexion logique ou une connexion empirique entre un énoncé et les conditions de sincérité de son énonciation? Pour répondre à cette dernière question, il faudrait savoir si la force illocutoire est syntaxiquement marquée dans l'énoncé. La force illocutoire assertive va de pair, logiquement, avec des conditions de sincérité associées. Si l'énoncé contient syntaxiquement une marque de la force illocutoire assertive, il implique une connexion logique avec les conditions de sincérité liées à son énonciation littérale. Or j'ai montré ailleurs que le mode indicatif était illocutoirement neutre. (Seymour 1985 ; voir aussi Récanati 1981) J'ai laissé entendre tantôt que, d'une façon générale, les énoncés d'attitudes propositionnelles à la première personne avaient des conditions d'assertabilité ambiguës. Cette thèse va dans le même sens que celle concernant le caractère illocutoirement neutre du mode indicatif. Dans le cas qui nous occupe, il n'y a pas d'ambiguité provoquée par deux usages positivement autorisés du verbe psychologique. Il 
s'agit plutôt d'invoquer cette fois-ci le caractère illocutoirement neutre du mode indicatif. Il y a certainement quelque chose de paradoxal à affirmer un énoncé et à nier ensuite les conditions de sincérité associées à son énonciation. C'est là l'essentiel du paradoxe de Moore. On ne peut pas affirmer «il pleut mais je ne crois pas qu'il pleut». Ma thèse est seulement que le paradoxe est pragmatique et non logique. J'en conclus que la connexion entre un énoncé et les conditions de sincérité de son énonciation littérale est empirique et non logique. L'énoncé (18) ne peut donc faire l'objet d'un savoir a priori.

Le cartésien peut en désespoir de cause répliquer que le même contenu de pensée est présent dans tous les actes accomplis en conformité avec la signification littérale de l'énoncé. Quand j'utilise les énoncés (12), (13), (14) et (18), j'exprime à chaque fois la pensée que je suis en train de penser. Il devrait donc y avoir une connexion logique entre l'énoncé et la pensée exprimée par son énonciation. Malheureusement, la connexion logique est entre un certain acte et la pensée qu'il exprime et non entre un contenu intentionnel (public ou mental) et la pensée qui s'exprime à l'occasion de son occurrence. L'agent sait sans doute $a$ priori que la seule occurrence de "Je pense * au sein d'un acte intentionnel de pensée entraîne la satisfaction de ses conditions de vérité, et ce, quel que soit l'acte (mental ou linguistique) accompli. Mais l'agent ne peut savoir a priori qu'une occurrence particulière constitue d'emblée l'occurrence d'un acte intentionnel de pensée. Pour qu'il puisse y avoir un tel savoir a priori, il faudrait que n'importe quelle inscription de la forme verbale (mentale ou publique) «je pense» puisse être considérée comme un acte d'exprimer la pensée que suis en train de penser. Or une inscription de cette forme verbale peut n'être rien d'autre que la réalisation d'un état fonctionnel et ne pas constituer, par conséquent, un acte intentionnel de pensée.

La distinction que je propose équivaut à celle que Davidson cherche à opérer entre les descriptions intensionnelle et extensionnelle de l'acte de tenir un énoncé pour vrai ("to hold true"). Il y a des lectures intensionnelle et extensionnelle de la phrase "A tient "p" pour vrai». Cette distinction est une instance particulière de la distinction générale que j'ai déjà proposée entre les usages intentionnel et matériel des verbes d'attitudes propositionnelles. Elle correspond aussi à une distinction plus traditionnelle entre les lectures "opaque" et "transparente* des verbes d'atti- 
tudes, pourvu que cette dernière ne soit pas interprétée seulement comme une distinction entre l'usage de dicto et de re. Le problème est que même si l'énoncé satisfait la condition sémantique et sert à rapporter un phénomène intentionnel et non seulement un état fonctionnel, on ne peut savoir a priori que la réalisation cognitive de l'énoncé équivaut à la performance d'un acte intentionnel. Par conséquent, l'agent ne peut savoir a priori que les conditions de vérité de l'énoncé qu'il utilise sont satisfaites par sa seule réalisation cognitive. Encore une fois, la raison est qu'il ne peut savoir a priori que toute inscription de l'énoncé constitue un acte qui exprime une pensée. Il est à noter qu'il suffit, pour réfuter l'argument cartésien, d'admettre la possibilité logique qu'une seule de ces inscriptions ne soit pas intentionnelle. Car on ne peut savoir a priori dans quel monde possible on se trouve. On ne peut exclure a priori l'éventualité que le monde dans lequel on se trouve soit précisément celui dans lequel n'est réalisé qu'un état fonctionnel.

L'argument que je viens de présenter implique en fait seulement qu'il n'existe pas une connaissance de soi qui est à la fois a priori et infaillible et qui se fonde sur la transparence épistémique. Cela n'exclut pas la possibilité de produire d'autres arguments a priori pour expliquer l'autorité de la première personne. J'ai aussi pris pour acquis qu'un savoir a posteriori ne pourrait pas se réclamer de l'infaillibilité. Mais cela reste à voir. Il y a peut-être là une porte de secours pour le partisan de l'infaillibilité. J'ai seulement voulu ici m'en prendre à un argument de type cartésien.

Département de philosopbie

Université de Montréal

\section{RÉFÉRENCES}

armstrong, David M. (1979), «Perception, Sense Data and Causality», dans Macdonald, G.F. (éd.) (1979), 84-98.

BURGE, Tyler (1988), "Individualism and Self-knowledge*, Journal of Philosophy, LXXXV, 11, 649-663.

DAVIDSON, Donald (1987), "Knowing One's Own Mind», Proceedings of the APA, 441-458.

DAvidson, Donald (1988), "Reply to Burge", Journal of Philosophy, LXXXV, 11, 664-665. 
DESCARTES, René, (1964), Deuxtème méditation, édité par C. Adam et P. Tannery, Oeuvres de Descartes, Paris, Vrin.

DUMmeTt, Michael (1979), "Common Sense and Physics*, dans Macdonald G.F. (éd.) (1979), 1-40.

gOOdman, Nelson (1951), The Structure of Appearance, Dordrecht, Reidel.

KRIPKE, Saul (1982), Wittgenstein on Rules and Private Language, Cambridge, Harvard University Press.

LEWIS, David (1979), «Attitudes De Dicto and De Se *, Philosophical Review, 88, 513-543.

LOCKE, John (1959), An Essay concerning Human Understanding, édité par Alexander Campbell Fraser, New York, Dover.

MaCdonald, Graham F. (éd.) (1979), Perception and Identity, Ithaca, Cornell University Press.

MCDOWELL, John (1984), «De Re Senses» Pbilosopbical Quarterly, XXXIV, 283-294.

MILLIKAN, Ruth (1984), Language, Thought and Other Biological Categories, MIT Press, Bradford Books, Cambridge, Mass.

PEACOCKE, Christopher (1981), "Are Vague Predicates Incoherent?», Syntbese, 121-141.

RÉCANATI, François (1981), Les énoncés performatifs, Paris, Les Editions de Minuit.

SEYMOUR, Michel (1985), * La force illocutionnaire est-elle une composante essentielle de la signification conventionnelle?» Dialogue, XXIV, 455-471.

WITtgenstein, Ludwig, (1953), Philosophical Investigations, Oxford, Basil Blackwell.

WRIGHT, Crispin (1975), «On the Coherence of Vague Predicates», Synthese, 325-365. 